\title{
Contrast Enhancement in Low-kV Zero-loss Filtered Imaging of Frozen-hydrated Biological Specimen
}

\author{
E Majorovits $^{1}$, G Hofhaus ${ }^{2}$, I Angert ${ }^{1}$, G Benner $^{1}$, U Kaiser $^{3}$ and RR Schröder $^{2}$ \\ 1. Carl Zeiss Microscopy GmbH, Oberkochen, Germany \\ 2. CellNetworks, Bioquant, Universität Heidelberg, Heidelberg, Germany \\ 3. Universität Ulm, SALVE Project, Ulm, Germany
}

Frozen-hydrated biological specimen yield low object contrast in conventional TEM images recorded typically at electron energies of $200-300 \mathrm{keV}$. This property is inherent to weak phase objects such as ice-embedded biological or soft matter material. In addition, the contrast and thereby the attainable resolution are limited by the characteristic electron dose that the specimen can tolerate before its structure is irrevocably destroyed. However, improved image contrast is crucial when visualizing cryospecimen e.g. for alignment procedures used in single particle analysis to obtain 3D structural information of biological macromolecules.

The conventional method to improve image contrast is defocusing. However, defocusing leads to information gaps in the contrast transfer function and in general to attenuated high resolution.. Another emerging technique is the use of phase plates [1], which should yield optimal contrast transfer but lacks practicability so far [2]. Here we investigate the contrast enhancement obtained by zero-loss filtering [3] combined with imaging at low electron energies.

We used Cs-corrected Energy Filtering TEMs (Zeiss LIBRA200 Cs) equipped with TVIPS TemCam F416 cameras to measure the improvement of image contrast with decreasing electron energy. Figure 1 shows a pair of zero-loss filtered images of TMV embedded in vitrified ice recorded at $40 \mathrm{kV}$ (A) and at $80 \mathrm{kV}$ (B) in focus. The applied electron dose was $2,5 \mathrm{e}^{-} / \AA^{2}$ and $3,8 \mathrm{e}^{-} / \AA^{2}$, respectively, and the ice thickness was determined to be about $40 \mathrm{~nm}$ in both cases. The image acquired at $40 \mathrm{kV}$ clearly shows better image contrast despite the lower applied electron dose. This is confirmed quantitatively by extracting profiles across the TMV strands and determining the contrast $(C=\Delta \mathrm{I} / \mathrm{I})$ : at $40 \mathrm{kV}$ the contrast equals $9,5 \%$ (figure $1 \mathrm{C}$ ) while at $80 \mathrm{kV}$ the contrast decreases to $4,9 \%$ (figure 1D). The analysis of a whole set of images of TMV recorded at electron energies of 20, 40, 60 and $80 \mathrm{kV}$ is shown in figure 2. The graph shows averaged contrast measurements in dependence of electron energy for zero-loss filtered (blue markers) and unfiltered (red markers) images. The energy dependence can be nicely fitted with a function proportional to the scattering cross section $\sigma: C \sim \sigma \sim 1 / \mathrm{v}^{2}$ ( $\mathrm{v}=$ electron speed). Furthermore, zero-loss filtering yields a contrast improvement be a factor of two for all examined electron energies, as expected from previous work [3].

The gain in contrast has to be seen in context with the applicable electron dose $\mathrm{D}_{\mathrm{e}}$. Our data confirm that beam damage is inversely proportional to the scattering cross section, $\mathrm{D}_{\mathrm{e}} \sim 1 / \sigma \sim \mathrm{v}^{2}$ (data not shown). Since the dose limited resolution $\delta$ is proportional to $\delta \sim \mathrm{C}^{-1} \mathrm{D}_{\mathrm{e}}^{-1 / 2} \sim \mathrm{v}$, the attainable resolution for typical cryo-specimen should increase with decreasing electron energy. First results show a trend in this direction (data not shown). However, a competing effect is the increasing contribution of inelastic scattering in a sample of finite thickness at lower electron energy (e.g. 20kV), which leads to a reduced zero-loss signal. In this case the electron dose limited resolution is further constrained by the thickness of the ice-embedded specimen [4]. 


\section{References:}

[1] E Majorovits et al, Ultramicroscopy 107 (2007) p. 213.

[2] R Danev, RF Glaeser, K Nagayama, Ultramicroscopy 109 (2009) p. 312.

[3] I Angert, E Majorovits, RR Schröder, Ultramicroscopy 81 (2000) p. 203.

[4] cf also: RF Egerton, European Microscopy Conference 2012, PS2.5, The importance of dose limited resolution.
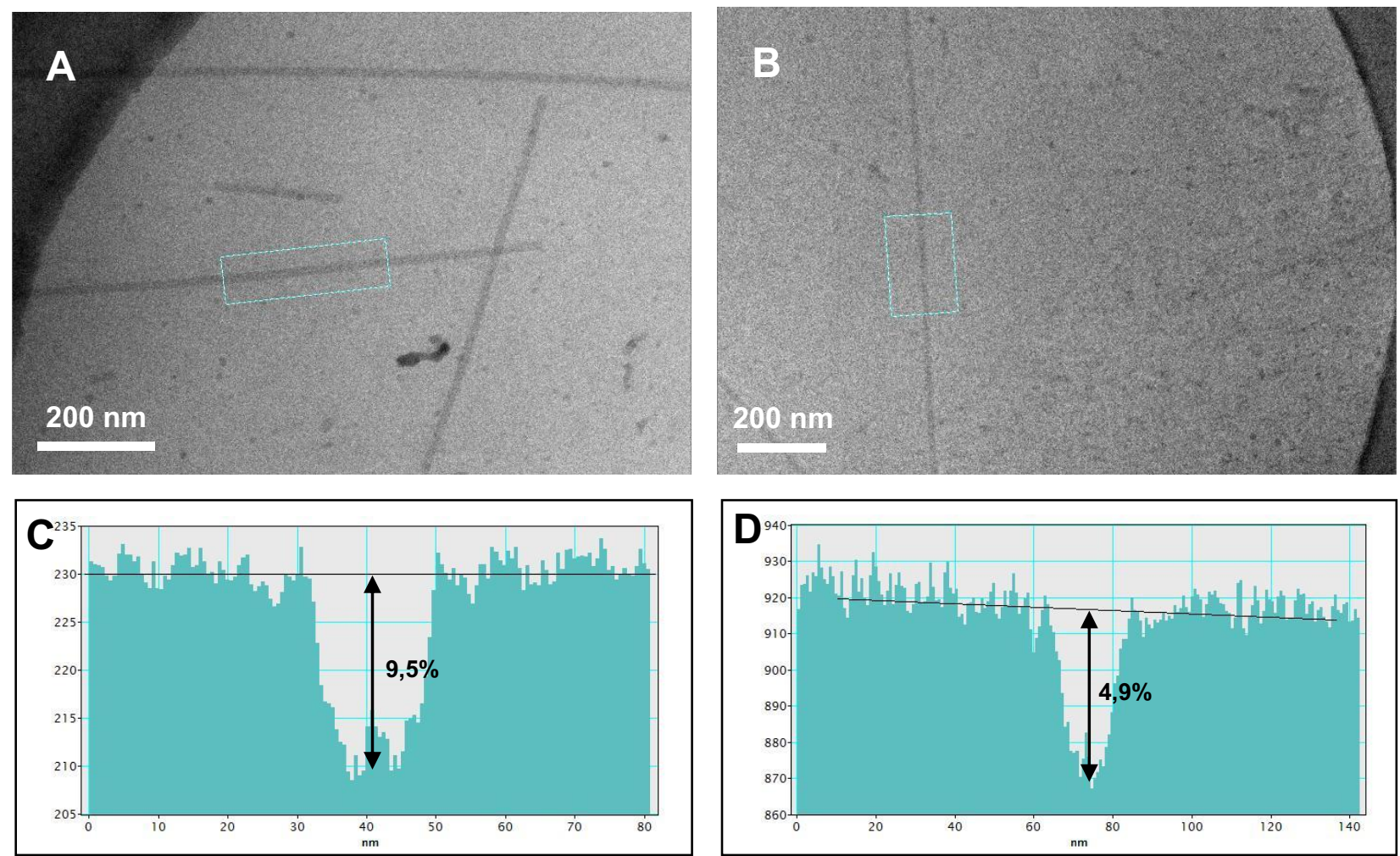

Figure 1. Zero-loss filtered images of TMV in vitreous ice recorded in focus at $40 \mathrm{kV}(\mathrm{A})$ and $80 \mathrm{kV}$ (B). The profiles across the TMV strand show the increased contrast at $40 \mathrm{kV}$ (C) versus $80 \mathrm{kV}$ (D).

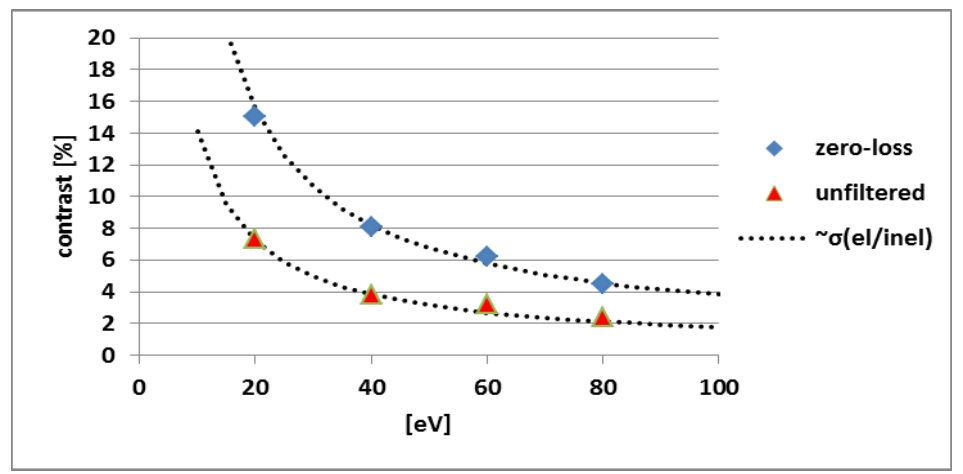

Figure 2. Image contrast in dependence of electron energy as determined from TMV profiles obtained from unfiltered (red) and zero-loss (blue) filtered images at various electron energies (see figure 1). 